\title{
Warum galten Epilepsie und Geisteskrankheit (Frenesis) als ansteckend? \\ Von
}

Dr. med. Alfred Martin in Bad-Nauheim.

„Febris acuta, ptisis, pedicon, scabies, sacer ignis, Antrax, lippa, lepra, nobis contagia praestant."

Diese Verse kennt Bernhard Gordon 1307 als einen Bestandteil des sog. „Regimen sanitatis Salernitanum“, das im Laufe der Zeit ständig an Umfang zugenommen hatte und weiter zunahm. Er ist der erste, der sie anführt.

Inhaltlich entspricht ihnen ein Eintrag im Baseler Ratsbuch, der zwischen 1.360 und 1404 erfolgte:

,Wele siechtagen ze schühende sient vnde wele lute, die" semlich siechtagen hand, [man] von der Stat triben sol.

Der erste siechtag ist eine durchspitzige suchte, Als mit den Büllen loufft.

Der ander siechtage ist die kurtzen Atem, als die lïte habent, den die lunge in die kelen gat oder wachset.

Der dritte siechtag ist der vallende siechtage.

Der vierte siechtage ist die stiebende rude. Der fünft siechtage ist sant Anthonyen rahe. Der sechste siechtage ist gifftige geswere. Der sibende siechtage ist ougen geswer. Der Achtoste siechtage ist miselsü.chtig oder veltsiech.

Vnd wer der Acht siechtagen einen hat, den sol man kein äsige noch trinkende dinge veil laßen haben vnd wie wol sie, das die heilige geschrifte nüt enthat, das man si alle von der welte scheiden sölle, So sint si doch alle ze schühende, wond si gand eins von dem Andern an. Vnd sol man die selben lüte, wo man die weisz, von der Stat heißen gan, vmb das die anderen, die gesunt sint, nyt den selben gebresten entphachent."

Sudhoff (1), der diese angebliche, zum Teil unverständlichẻ Baseler Ratsverordnung durch Witteilung der Gordonschen lateinischen Verse $k$ ]ärte, bezweifelt die Rechtsgeltung derselben. Ich glaube, es handelt sich 
schon wegen der Nichtdatierung um einen Entwurf, der mit seinem Stadtverweise nur für Landfremde berechnet sein konnte.

Die durchspitzige Suchte $=$ sehr akute $($ durch $=$ sehr, spitzig $=$ akut) fieberhafte Erkrankung, die mit den Büllen, d. i. Beulen einhergeht, ist die Pest, die also unter Febris acuta zu verstehen ist. 2 ist Phthisis = Lungenschwindsucht, 4 Scabies, womit nicht nur unsere heutige Scabies (Milbenkrätze), sondern das, was das Volk Raude nennt, gemeint ist, 5 Sacer ignis ist St. Antonien Rahe (Rache) oder Feuer $=$ Erysipel, Phlegmone und Brand, 6 Anthrax $=$ Karbunkel mit Einschluß des Milzbrandkarbunkels, 7 sind schwere Augenerkrankungen mit starker Sekretion und Borkenbildung unter Einschluß des Trachoms und der Gonorrhöe, 8 ist Aussatz (die Krankheit, welche nach der heiligen Schrift das Scheiden von der Welt, d. h. die Ausstoßung aus der bürgerlichen Gesellschaft verlangt).

Sudhoff (1) erklärt die Krankheiten als: Beulenpest (könnte, zwar nicht in dem Baseler Falle, auch Febris pestilentialis, Flecktyphus sein), Phthisis, Skabies, Erysipel, Milzbrand, Trachom, Lepra.

Ich habe oben auf Grund meiner Studien (2) die Begriffe weiter gefaßt. Wir sehen zwar, daß einzelne der genannten Krankheiten ansteckend sind und daß in den Krankheitsgruppen ein oder zwei ansteckende Krankheiten enthalten sind, die Naturbeobachtung war aber doch nicht so weit gediehen, um aus ähnlich verlaufenden Krankheiten die infektiösen herauszukennen, z. B. bei der lippa, oder aus einer Gruppe von Krankheiten, die trotz ihrer großen Verschiedenheit als eine aufgefaßt wurden (das Antoniusfeuer), die infektiöse Komponente (hier das Erysipel) auszusondern. Am meisten wird die ungenügende Naturbeobachtung durch die Aufnahme von Nr. 3 in das Verzeichnis bewiesen, des fallenden Siechtages, d. i. der Epilepsie, die im lateinischen Vers den Namen Pedic on führt.

Der Name Pedicon ist bisher ethymologisch nicht aufgeklärt; ich finde, daß er frühzeitig nicht mehr verstanden wird. Andere Verse haben dafür Morbus caducus.:

„Nota quinque sunt morbi contagiosi unde versus:

Lepra, febris, lippa, scabies, morbusque caducus

Corporibus nostris transicione nocent."

Wir sehen hier, aus einer Handschrift der Breslauer Universitätsbibliothek aus der zweiten Hälfte des 14. Jahrhunderts, nur fünf ansteckende Krankheiten angeführt. Sudhoff (3) macht gelegentlich dieser Veröffentlichung darauf aufmerksam, daß Pedicon hier mit 
Morbus caducus wiedergegeben ist, wie es allgemein verstanden wird, trotzdem sich einem immer wieder die Vermutung aufdrängt, es müsse mit Impetigo lautlich zusammenhängen, also eine ansteckende Krankheit parallel zur Scabies bezeichnen. Anderseits bilde die historische Tatsache der Ausschließung der Epileptiker von den römischen Komitien (daher Morbus comitialis) einen gedanklichen Übergang für die Einregistrierung der Fallsucht unter die sonstigen ,Morbi separandi".

Man sieht, daß hier Sudh off, wie schon oben, ron dem Gedanken beherrscht wird, daß nur wirklich ansteckende Krankheiten in die Merkverse aufgenommen wurden, wobei er die Naturbeobachtung der Alten überschätzt. Übrigens ist in dém verhältnismäßig reichen Stoff nur einmal, in der oben angeführten sog., nicht einmal gültigen Baseler Ratsverordnung von Absonderung die Rede, sonst nur von der Ansteckung der Krankheit und dem Scheuen, dem Fliehen vor ihr; auch in Rom wurden ja die Epileptiker nicht abgesondert, Morbus contagiosus und Morbus separandus deckt sich eben nicht.

Fine mittelalterliche deutsche Utbersetzung der Wiener Hofbibliothek (Ms. 2864) gibt Bernhard Gordons pedicon gar mit fuz gestanch wieder (4), wobei der Anklang an pes das nicht mehr geläufige Wort erklären mußte.

Warum die Krankheiten ansteckend sind, beantwortet Bernhard Gordon: „Materia incorrigibilis et inrectificabilis generat morbum hereditarium et interficit se et vicinum suum ${ }^{\text {, }}$, was die eben erwähnte Wiener Handschrift übersetzt: "Vnd do von ein isleich vnrayn materia, dy man nicht richten vnd recht gepezzern mag, de gepurd in erb seuch vnd furunraynet sich an ein ander." (4) Hierbei sei bemerkt, daß Morbus hereditarius und daB die entsprechende Erbseuche keine erbliche, sondern eine ansteckende Krankheit ist, heute noch erbt man im Alamannischen eine Krankheit, d. h. wird mit ihr angesteckt.

,Si gand eins von dem Anderen an," heißt es im Baseler Rats: buch, also ein Mensch bekommt es vom andern. "Sie chöment von ainem zu dem andern" hat ähnlich Cod, germ. 317 [wohl der Münchener Staatsbibliothek]. Und weiter heißt es hier: ,vnrain prodem, der von den lewten get vad von pösem gestankch, wan $[=$ denn $]$ die lüft treibt das von ainem czu dem andern vnd veecht den menschen an, dauon er auch chümpt in dise siechtum ${ }^{6}(5)$.

Ich möchte hier nicht, wie Sudhoff (5), das Durchschlagen der älteren griechischen Idee dex Luftverderbnis, des Miasmas sehen. Materiell 
wird die Krankheit durch Atem und Ausdünstung des Körpers übertragen, von Mensch zu Mensch, durch die Luft hindurch. Nicht die Luft wird verdorben und wirkt nun krankheitserregend, sie befördert nur den Krankheitsstoff, der für jede Krankheit spezifisch angenommen sein muß.

Mit dieser Anschauung hängt zusammen, wenn der Deutschböhme Siegmund Albich 1484 in seinem ,Tractatulus de regimine hominis" (die Stelle entnehme ich Sudhoff (4)) sagt: ,,igitur non loquimini cum eis nec balneamini, quia solo anhelitu inficiunt hominem, " daher sprecht und bädet nicht mit ihnen, weil sie durch die Ausdünstung allein den Menschen anstecken, wobei daran zu erinnern ist, daß das damalige Reinigungsbad ein Schwitzbad war.

Wie kommt die Epilepsie unter die auf diese Weise ansteckenden Krankheiten? Die Auskunft gibt uns eine Predigt (6) des 1272 gestorbenen Minoriten Berthold von Regensburg:

„Noch sint etlîche siechtüeme die der sieche hât. Sumelîche liute hânt den siechtuom, den alle meister niht vertrîben künnent; unde giengen alle meister zuo, die von erznîe ie gelâsen, die künden etelîchen siechtuom niemer vertrîben noch gebüezen [= heilen]. Unde lebete meister Ypocras [Hippolzrates] noch hiute, der meister was über alle meister die von erzenîe ie gelâsen, er kündez niemer gebüezen, unde lebte noch her Galiemus unde her Constantînus [Africanus] unde her Avicennâ unde her Macer und her Bartholomêus, - die wâren die aller hôhesten meister die von erznîe ie gelâsen, unde hạbent alle künste erfunden und erdât, diu von erznîe ie wart erdâht - , unde lebten die alle noch, sie möhten etelîchen siechtuom niemer gebüezen. Daz ist ûzșetzikeit und ist diu vallende, suht. Swer die vallende suht hât über vier unde zweinzic jâr, dâ gên alle die zuo die dâ hiute leben, die künden den siechtum niemer gebüezen. Unde swenne er alsô hin vellet unde lît unde schûmet, sô hüetet iuch vor im als liep iu lîp si, daz sich ieman nâhen zuo im habe, wan im gêt ein sô griulich âtem ûz dem munde, daz er vil lîhte den selben siechtuom gewünne, swem der âtem in den munt kaeme. Unde dâ von sô hüetet iuch daz ir im iht nâhen komet innen des, daz in der siechtuom an gêt."

In unserem heutigen Deutsch heißt der letzte Abschnitt ungefähr: Und wenn er hinfällt und liegt und, schäumet, so hütet euch vor ihm, so lieb euch euer Leib ist, daß jemand in seine Nähe komme, denn ihm geht ein so gräulicher Atem aus dem Munde, daß er sehr leicht dieselbe Krankheit bekommen kann, wenn der Atem in seinen Mlund geht. Darum hütet euch, daß ihr ihm nicht nahe kommt, währenddessen ihn die Krankheit befällt. 
Als Krankheit wird also der epileptische Anfall betrachtet, und nur in diesem steckt der Kranke an, wobei man annimmt, daß sein aufschäumender Speichel den Krankheitsstoff ausdunstet. Der Schaum vor dem Mund im Anfall gab demnach die Ursache dafür ab, daß die Epilepsie unter die ansteckenden Krankheiten eingereiht wurde.

Aus Bertholds Predigt ersehen wir auch, daB die Epilepsie für die schwerste unheilbare Krankheit neben dem Aussatz gehalten wird ), deren Ansteckung man demnach ganz besonders fürchten mußte. Das tat man auch.

Oben wurde schon erwähnt, daß nach Sudhoff (3) die Epileptiker von den römischen Komitien ausgeschlossen wurden ). Bei den Zisterziensern war außer dem Aussatz in alter Zeit auch Epilepsie ein Hindernis für die Aufnahme in den Orden (8).

Im 16. Jahrhundert kommt als dritte unheilbare Krankheit die Syphilis hinzu. In einem Gedicht eines ungenannten Ritters aus Franken, das 1537 erschien, rühmt sich das Podagra, daß es die Menschen nicht so plagt wie andere Krankheiten, „Die on vnderlaß vnd vnfrist Peinigen, wie dan thůt Mentagra (Syphilis) Sanct Veltins kranckheyt (St. Valentins Krankheit = fallende Sucht) vnd lepra, Die sie khein artznei lond zwingen Vnd krank in verzweyflung bringen" (9). Dem entspricht, wenn es im Stiftungsbrief des Zwölfbruderhauses in Nürnberg vom 12. Januar 1510 heißt: „Ob aber der bruder ainer mit dem awßsatz, dem hinfallenden siechtagen oder Krankheit der Frantzose $n$ begriffen wurd, der sol bey den andern Brudern nit gelitten, sonnder zu stund gevrelawbt [entlassen] werden" (Murrs Journal für Kunst und Lit. Th. 10) (9).

Die Epilepsie war demnach unter den ansteckenden Krankheiten neben dem Aussatz und später auch noch neben der Syphilis am gefürchtesten.

1) Er beruft sich dabei auf die Meister der Arznei und nennt Namen ron gutem Klang verschiedenster Richtung. Er war demnach nicht der ungebildete, die Wissenschaft verachtende Volksredner, als den mán ihn gern hinstellt. Der von mir gesperrtgedruckte Abschnitt ist ohne Hinweis auf einen Meister der Arznei und enthält seine Ansicht, die wohl der Volksmeinung der Zeit entspricht, wobei ich nicht in Abrede stellen will, daß diese der Schulmedizin entnommen ist.

2) Heller (7) sagt allerdings, daß die Epilepsie nach Celsus Komitialkrankheit heißt, weil die Komitien unterbrochen wurden, sobald der Kranke an diesem Tage von einem Anfall heimgesucht wurde, denn man deutete dies als ungünstiges Zeichen. 
Wie man sich in der 1. Hälfte des 16. Jahrhunderts die Entstehung des epileptischen Anfalls auf Grund der Tradition dachte, zeigt uns Johann Agricola (10) bei der Firklärung des Fluches: ,Das dich die plag bestehe“. Er sagt 1530: „Die plage ist ... das Falbel, das Fallend ubel, die große seüche, die große kranckhait. Dyse plag ist also gethan, wie Hypocras schreibt, das, wenn sie vntex Fünff vnd zwaintzig jaren bicht vergehe, dem weret sie biß an sein ende, yhr ankunfft, ist ein böser tampff vnd ein gyfft die einer hat ym magen, etliche auch ynn einer zehen, wenn sich nun der tampff vnd die gifft erhebt, so wirdt das hirn tropffen, vnd sie müssen fallen." (Es sei eingefügt, daß das Niederstürzen beim Schlaganfall nach ein er Meinung der Alten auch durch das Fal] en eines Tropfens vom Gehirn erzeugt wird, fällt der rechte Tropfen kommt rechțs die Lähmung, auf der linken Seite liegen die Verhältnisse ebenso, fallen beide, gibt es den Tod.)

Agricola hat sich über die Ursache aber auch eine eigene Meinung gebildet. Beim Fluch: „Das falbel gehe dich an" erklärt er: ,Also reden die Sachsen vnnd Döringer, sonst solle es hayssen daz fallend übel, morbus comitialis. Denn die lewtte, welche hie mit beschwerdt sind, fallen gemainiglich wo vil lewte sind [er begründet hier also den Namen morbus comitialis und gibt jedenfalls damit die beste Erklärung], vielleicht von dem brunst vnd, athem viler lewtte."

Wir sehen, daß man anfing, sich von der Tradition loszumachen und zu beobachten. Nicht mehr das aus dem Magen oder den Zehen aufsteigende dampfende Gift ruft den Anfall hervor, und damit war die Unterlage gegeben, dieses Gift auch nicht mehr im Nundschaum zu sehen, wodurch die Epilepsie aus der Reihe der ansteckenden Krankheiten folgerichtig ausscheiden mußte.

Zum Schluß seien noch 2 Laienbeobachtungen angeführt. Der Straßburger Münster-Prediger Geiler von Kaisersberg (11) sagt 1508 in einer Predigt: „Also geht es denen die sant Veltins siechtagen hond wen sie den siechtagen leiden so entpfinden sie nit, waz man inen anthüt". Im Wunderbuch der Wallfahrtskirche in Grimmenthal in Thüringen (12) findet sich am 27. Mai 1514 der Eintrag: , . . . ist hy erschynen ein man von Freybergk, Valentin Clemen genannt, des weib in kindsnoten gelegen ist, in welchen noeten $d y$ grossen krankheit sant Valtins sein weib zwengzik mal gepeiniget hat, ist von ym ein gelubnis gescheen Mariam im Grymtal zu besuchen mit $2 \mathrm{lb}$. wachs. Alsbald hat solche crankheit sein weib genzlich verlassen, ein schons liblich kindt geboren und kurzlich gesunth worden."

Hier wird die große Krankheit Sankt Valentins geheilt, sie ist in diesem Falle aber Eklampsie. Zwei weitere Fälle von Heilungen, die verzeichnet sind, müssen wir als Hysterie ansprechen, in dem einen hatte der Kranke von Petri kathedra bis Margarethe alle Tage einen Anfall gehabt.

Auch die Geisteskrankheiten wurden zu den ansteckenden Krankheiten gerechnet. 
,Febris acuta, ptisis, pedicon, scabies, sacer ignis, Antrax, lipa, lepra, frenesis contagia prebent."

heißt es in der Karlsruher St. Georgenhandschrift LXI, die nach Sudh off kurz nach 1300 geschrieben zu sein scheint, und ebenso in M. 2002 der Gymnasialbibliothek in Heilbronn aus der Mitte des 15. Jahrhunderts (13). Frenesis heißt die 9. Krankheit. Sudhoff sagt eimmal (1), daß er das frenesis der Vulgata des Regimen sanitatis für eine spätere Zutat oder Contaminatio halte. Das entspricht seinem oben angegebenen Standpunkt, daß nur wirlklich ansteckende Krankheiten in das Verzeichnis aufgenommen wurden.

Der erwähnte Siegmund Albich (4) erklärt 1484: „Frenesis, id es demencia, vnsyunnykeyt adir toricht." Cod. german. 317 (5) hat: ,die vnsinnig sind“. Der Baseler Vocabularius optimus (14) aus dem 14. Jahrhundert bringt ,Frenesis Hovpt geswer", und Hans von Gersdorf (15) sagt 1517 in dem seinem Feldbuch der Wundarznei angehängtem Vocabularius Infirmitatum: „Frenesis hirnwuty, hirndobigkkeit ist ein geschwer am vorderen teyl des hirns."

Daß Hans von Gersdorf einer Gehirnsektion (die er abbildet) beigewohnt hat, ist anzunehmen, kaum aber, daß er bei einem Geisteskranken grobe Veränderungen im Stirnhirn gesehen hat. Auch der Baselér Vocabularius nimmt ja schon - sicher rein theoretisch" - ein Hauptgeschwür an. Da Lungengeschwür, wie die Lungenschwindsucht auch genannt wird, giftig Geschwär (Anthrax) ansteckend sind, hat man auch das Hauptgeschwür (Geisteskrankheit) dafür gehalten.

Man muß aber bedenken, daß die Tollw ut (Hundswut) als schwere Geisteskrankheit galt. Eines der Hauptgegenmittel und zwar auch bei schon ausgebrochener Wut war die Anagallis arvensis, der Gauchheil oder Geckenheil = Heilmittel der Toren, der Narren (16).

Geht man noch weiter zurück, trifft man in den ,Lecciones Heliodori" (17) (die dem zur Zeit Trajans, lebenden Chirurgen Heliodoros zugeschrieben werden (18)) die auch beim Pseudo-Soran und PseudoGalen vorkommende Stelle: ,Frenesis dicitur alienaceo cum acutis febribus periculum concitans": Das wären gefährliche fieberhafte geistige Störungen, bei denen an erster Stelle an das Nervenfieber, den Typhus gedacht werden muß.

Wir haben hier eine Parallele zum. Antoniusfeuer, bei dem ein Bestandteil, das Erysipel, ansteckend ist und eine andere, mit darunter begriffene Krankheit, der Brand, auch ansteckend sein mußte. 
Das Nervenfieber (Typhus) steckt an, folglich waren alle anderen Geisteskrankheiten auch ansteckend, und weil sie ansteckend waren, schloß man, daß ihnen ein Hauptgeschwür zugrunde liege.

\section{Literatur.}

1. Sudhoff, Karl, Die acht ansteckenden Krankheiten einer angeblichen Baseler Ratsverordnung vom Jahre 1350. Wiener med. Wochenschr. 1913.

2. Martin, Alfred, Der Pestteufel in der Versuchung des hl. Antonius auf dem Isenheimer Altargemälde von Matthias Grünewald. Münchn. med. Wochenschr. 1920, Nr. 34. Eine weitere diesbezügliche Arbeit erscheint demnächst in der Schweiz. med. Wochenschr.

3. Sudhoff Karl, Fünf ansteckende Krankheiten. Mitteilungen z. Gesch. d. Med. u. Naturwissenschaften. XIV. Bd., Nr. 5.

4. Sudhoff, Karl, Ein spätmittelalterliches Epileptikerheim zu Rufach im Oberelsal3. Arch. 1. Gesch. d. Med. Bd. VI, H. 6.

5. Sudhoff, Karl, Welche Menschen man fliehen soll. Ebenda. Bd. VIII, H. 2 u. 3.

6. Pfeiffer, Franz, Berthold von Regensburg. Vollständige Angabe seiner Predigten. 1. Bd. Wien 1862.

7. Heller, Frits, Über Pathologie und Therapie der Epilepsie im Altertum. Janus. 16. Jg. Fiarlem 1911.

8. Müller, Gregor, Von unseren Kranken. Cistercienser-Chronik. 1898, 10: Jg. 1898.

9. Fuchs, C. H., Die ältesten Schriftsteller über die Lustseuche in Deutschland von 1495 bis 1510 . Göttingen 1843 .

10. Agriccla, Johann, Das ander teyl gemainer Tewtscher Sprichwörter, nuit jhrer außlegung. Eisleben $\mathbf{1 5 3 0 .}$

11. Geiler von Keisers perg, Johannes, Die Emeis. (Straßburg 1517.)

12. Dersch, Wilhelm, Ein Wunderbuch der Wallfahrtskirche in Grimmenthal. Henneberger Blätter. (Sonntagsblatt der Dorfzeitung, Hildburghausen 1921.)

13. Sudhoff, Karl, Zum Regimen Sanitatis Salernitanum., Arch. f. Gesch. d. Med. Bd. XII, H. 5.u. 6.

14. Wackernagel, Wilhelm, Vocabularius optimus. (Basel) 1847.

15. (Meister Hans von GerBdorff, genant Schylhans), Feldtbüch der wundtartzney. Straßburg (1517).

16. Martin, Alfred, Geschichte der Tollwutbekämpfung in Deutschland. Hessische Blätter für Volkskunde. Bd. XIII.

17. Sigerist, Henry W., Die ,Lecciones Heliodori“". Archiv f. Gesch. d. Med. Bd. XIII, H. 5 u. 6.

18. Sigerist, Henry F., Die, „Cirurgia Eliodori“. Ebenda. Bd. XII, H. 1 u. 2 . 